\title{
Modeling the Interaction between a Thermal Flow and a Liquid: Review and Future Eulerian-Lagrangian Approaches
}

\author{
Stéphane Vincent ${ }^{1 *}$, Erick Meillot ${ }^{2}$, Céline Caruyer ${ }^{2,3}$, Jean-Paul Caltagirone ${ }^{3}$ \\ ${ }^{1}$ Université Paris-Est Marne la Vallée, Laboratoire MSME, UMR 8208, Champs-Sur-Marne, France \\ ${ }^{2}$ CEA-DAM, Le Ripault, F-37 260 Monts, France \\ ${ }^{3}$ Université de Bordeaux, Laboratoire I2M, Pessac Cedex, France \\ Email: *stephane.vincent@u-pem.fr
}

How to cite this paper: Vincent, S., Meillot, E., Caruyer, C. and Caltagirone, J.-P. (2018) Modeling the Interaction between a Thermal Flow and a Liquid: Review and Future Eulerian-Lagrangian Approaches. Open Journal of Fluid Dynamics, 8, 264-285. https://doi.org/10.4236/ojfd.2018.83017

Received: December 11, 2017

Accepted: July 16, 2018

Published: July 19, 2018

Copyright $\odot 2018$ by authors and Scientific Research Publishing Inc. This work is licensed under the Creative Commons Attribution International License (CC BY 4.0).

http://creativecommons.org/licenses/by/4.0/

\begin{abstract}
Suspension Plasma Spraying is a complex process in which several physical mechanisms play a part. So the modeling and understanding of the interaction between a high-velocity and thermal flow and a liquid precursor phase is of major importance concerning the control and characterization of the process. The liquid droplet size distribution has a high influence on the kinetic properties of the as-sprayed nanometer particles before impacting on a target substrate. An overview of existing models is provided dealing with the penetration of the liquid phase into the thermal flame and the resulting fragmentation and vaporization of this phase before impact. The physical characteristics of the flow as well as existing Lagrangian and Eulerian modeling strategies are briefly discussed while paying attention to the physical parameters characterized and measured by numerical simulation. The potential of the various models and also their limits are intended to be highlighted. Future coupled Eulerian-Lagrangian modeling strategies are also proposed for a global and more exhaustive representation of the injection, fragmentation and dispersion part of the two-phase gas-liquid flow before particle impact on the substrate.
\end{abstract}

\section{Keywords}

Modeling, Liquid/Plasma Interaction, Fragmentation, Thermal Spraying Simulation

\section{Introduction}

Increasing higher efficiency rates or lifetimes of functional industrial parts re- 
quires the development of new materials such as ceramic coating. For many years now, the industry has relied upon surface treatment processes to improve them by numerous materials deposition. In the ceramic deposition field, plasma spraying or High Velocity Oxy Fuel processes are the main one to deposit ceramics. Since the end of the 1990's, the processing of nanostructured materials has been performed using them [1] [2] [3] [4]. This scale field, in which can be defined the characteristic length of the different physical processes (electronic, mechanical, optical...) and in which the structure organization plays a main role in these processes, confers to the type of coatings specific increased properties. By thermal flow, the High Velocity Oxygen Fuel (HVOF) [5] and plasma processes are concerned in order to heat and accelerate nanoparticle of ceramic materials [6]. Heat sources such as these techniques are required due to the high heat level to be transferred to the ceramic material. For example, Cold spray process is not adapted due to its concept based on only acceleration by high gas flow rate.

Now, thermal spraying is up-to-date to build nanometre-scale structure. Nevertheless, modelling and simulation of the interaction between a thermal jet and a liquid precursor phase continue to be of major importance concerning the control and characterization in terms of size of

- Carrier liquid droplet (size, distribution, position and velocity),

- Distribution of nanometer particles before impact on a target substrate,

- And thermokinetic properties of these solid particles carried by the liquid phase. The typical problem of interest is illustrated in Figure 1 whatever process is: the main difference between the two processes being the plume generator and the direct injection of the liquid inside it. Each technique can produce coatings to provide protection against high temperatures, corrosion, erosion or wear on materials [7]. For plasma spraying gun, around a cylindrical cathode, the operating gas (either pure, e.g., argon, or, more commonly, a mixture, e.g., argon/hydrogen, argon/hydrogen/helium) enters the torch and is heated by the electrical arc generated between a nozzle-shaped anode (of oxygen-free copper) and the cathode. This results in the transformation of the gas into plasma, which exits the torch as a free jet. HVOF is an internal combustion system that generates a supersonic flame jet.

Decreasing the deposit width $(<100 \mu \mathrm{m})$ with nano-structured coatings leads to improve as-manufactured properties, but due to their low size and low inertia, nanometer particles are injected in the jet with a liquid precursor [8].

From a modelling point of view, several major informations can be extracted from this figure:

- the heat and mass transfers are multi-scale in time and space,

- the multi-physic characters of the flow as chemical, thermal, turbulent, multi-phase and electromagnetic features,

- The coupling between fluid and solid mechanics.

These mechanisms have to be modelled depending on the considered zone of the problem (from hot flame inlet to impact of particles). General reviews have 


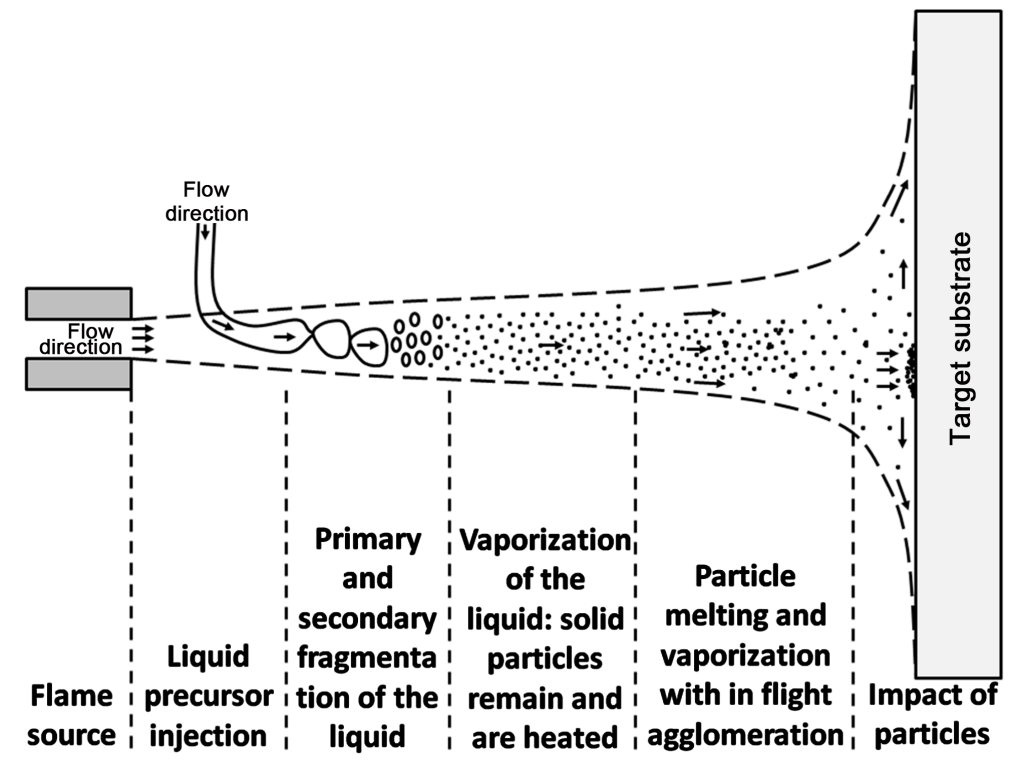

Figure 1. Interaction between a thermal flame and a suspension containing dense particles in a spraying process.

been published recently [9] [10] concerning both modelling or experiments for thermal spray processes.

Among the numerous contributions leading to models for suspension plasma spraying, those dealing with multi-phase heat and mass transfers can be classified in three categories:

- Experiments and macroscopic behaviour laws that provide a global description of specific parameters of the process. For example, the flow and thermal transfer characteristics have been widely measured for both HVOF and plasma gun configurations. Thanks to these experiments, the chemical composition of the gas as well as the temperatures or velocities of the related flow have been obtained by numerous authors [5] [11] [12]. In particular, the unsteady character of the plasma flow has been described by [13] [14] [15] [16]. Recently, high speed imaging, shadowgraph, Spray Watch and emission spectroscopy techniques provide a local description of liquid/plasma interaction [17] [18] leading to a better understanding of the coupling between the inlet flow and the liquid injection regimes. The droplet breakup regimes are governing by Weber, Ohnesorge and liquid Reynolds numbers. Classically, the Weber number $(\mathrm{We})$ is defined as the ratio of the disrupting aerodynamic forces to the restorative surface tension force. The Ohnesorge number represents the ratio of drop viscous forces to surface tension forces. When the Ohnesorge number is inferior to 0.1 , the transitions between different breakup modes depend only on the Weber number. The description of the different break-up modes according to the Weber number is reported in $\mathrm{Ta}$ ble 1 [19].

All these parameters are of primary importance when evaluating the type of liquid/flame interaction that will occur according to existing classifications of 
Table 1. Breakup modes according to the Weber number.

\begin{tabular}{cc}
\hline Breakup mode & Weber number \\
\hline Vibrational breakup & $W e<12$ \\
Bag breakup & $12<W e<50$ \\
Bag and Stamen breakup & $50<W e<100$ \\
Sheet stripping & $100<W e<350$ \\
Wave crest stripping & $W e<350$ \\
Catastrophic breakup & $W e>350$ \\
\hline
\end{tabular}

break-up phenomena [20]. In addition, other effects such as the vaporization of the liquid can be estimated using Nusselt convective laws [21] [22]. Other macroscopic laws for the liquid phase have been proposed [22] [23] [24] [25] [26] concerning the evolution of the liquid jet break-up in terms of the resulting droplet diameter (TAB or ETAB models) or temperature inside the thermal flow, based on measurements and theoretical developments. These global models have been used to predict the trajectories of droplets inside the flame [27]. In this work, the mean experimental velocity and temperature were utilized as inputs to the particle diameter and temperature models. For the purpose of flow characterization, experiments and constitutive models are the only way to obtain the transport coefficient such as density, viscosity, heat conductivity or specific heat [28] [29]. These coefficients are required to investigate local modelling and simulation. Another major interest of experiments and related phenomenological laws is validations of models and simulations:

- The heat and fluid flow models for the flame without liquid injection. Many works have been devoted to the modelling and simulation of the flame as a preliminary step to injection of powders or carrier liquid droplets. If reference is made to the Mach $\mathrm{Ma}(\mathrm{Ma}=\mathrm{V} / \mathrm{c}$ with $\mathrm{c}$ is the speed of sound in the medium) and Reynolds Re numbers $(\operatorname{Re}=(\rho v D) / \mu$ with $\mathrm{D}$ is the diameter of the torch exit) which belong respectively to the ranges $0.3 \leq \mathrm{Ma} \leq 2$ and 1500 $\leq \mathrm{Re} \leq 8000$ depending on the plasma and HVOF characteristics, it can be deduced that turbulence and compressible effects have to be taken into account. A majority of the flow models assumes that a continuum medium is representative of heat and mass transfers and that the flow is turbulent, compressible (except [26] [30] [31] who consider an incompressible flow) and can be modelled by the Random Average Navier-Stokes (RANS) equations [11] [15] [32] [33]. The energy conservation accounts for radiative effects through a local volume source term whereas the transport of species coming from the flame inlet are modelled by the resolution of all compounds [32] or by considering the ionized gas as a specific fluid [34]. With specific plasma conditions, leading to the restrike mode for example [35], the unsteadiness of the flow cannot be reproduced correctly by RANS approach [16] due to the multiple phenomena appearing in the flow. Recent works have introduced 
the use of Large Eddy Simulation (LES) for modelling the jet with success. The unsteady character of the thermal flow has been nicely recovered using LES turbulent modelling when compared to experiments [16] [36].

- The plume generator models: specific characters of this part of the suspension spraying process require the use of dedicated models for accounting of electromagnetic effects in a plasma torch [24] or of combustion effects in a HVOF gun [5] [10]. For the HVOF combustion, equations are in accordance with the combustive/fuel chemical reactions. For the plasma, several models have been proposed to simulate it, and their complexities evolve with increasing computational resources. The first simulations were limited to steady plasma flows and utilized temperature and velocity profiles as input data [12] [32] [37] [38]. The average result fields of the jet flowing though the ambient atmosphere do not take into account the transient plasma flow behavior which is becoming increasingly important for suspension spraying due to high frequency of the mechanisms appearing. Two routes can be employed for a non-stationary flow outside the torch:

- A global approach, integrating the physical phenomena through the distinct equations of electromagnetic and fluid mechanics. These studies employed the same set of equations, based on the mass, momentum and energy conservation. This was coupled with the Maxwell equations for electromagnetism effects based on the local thermodynamic equilibrium (LTE) assumption for the gases, which supposes that all the species are at the same temperature. Last recent works are from [39]-[44]. These approaches lead to a better understanding of the jet generation inside the torch.

- A simple approach based on a Joule effect in the arc column including correlations with experimental time-dependent voltage measurements [24] [45]. This approach leads to only investigate the unsteady flow outside the torch.

The turbulence models and energy conservation models are the same as those described previously.

The article provides an overview of existing models for dealing with the penetration and the transport of the liquid phase within a thermal flow and the resulting fragmentation and vaporization of this phase before impact on the target substrate. In the two first sections, existing Lagrangian and Eulerian modelling strategies are briefly discussed while paying attention to the physical characteristics obtained by numerical simulation. Comparisons with existing experiments are provided in order to highlight the potential of the various models and also their limits. The final part is devoted to future coupled Eulerian-Lagrangian modelling strategies for a global and more exhaustive representation of the injection, fragmentation and dispersion of the two phase flame-liquid flow before impact on the substrate. Conclusions are finally drawn.

\section{Lagrangian Models for Liquid Precursor Interaction with a Thermal Flow}

For both HVOF [5] [11] [46] [47] and plasma [15] [33] [48] thermal flows, the 
modeling of the interaction between the liquid precursor phase and the thermal flow has been mostly investigated by means of a RANS statistical representation of the turbulent effects and a compressible formulation of the momentum equations as follows:

$$
\begin{gathered}
\frac{\partial \rho}{\partial t}+\nabla \cdot(\rho \boldsymbol{u})=S_{m} \\
\rho\left[\frac{\partial \boldsymbol{u}}{\partial t}+\nabla \cdot(\boldsymbol{u} \otimes \boldsymbol{u})\right]=-\nabla p+\nabla \cdot\left(\mu+\mu_{t}\right)\left[\nabla \boldsymbol{u}+\nabla^{t} \boldsymbol{u}\right]+\boldsymbol{S}_{M} \\
\rho\left[\frac{\partial h}{\partial t}+\nabla \cdot(h \boldsymbol{u})\right]=\nabla \cdot\left(\lambda+\lambda_{t}\right) \nabla T+S_{h} \\
\rho\left[\frac{\partial \xi_{i}}{\partial t}+\nabla \cdot\left(\xi_{i} \boldsymbol{u}\right)\right]=\nabla \cdot\left(D_{i}+D_{i, t}\right) \nabla \xi_{i}+S_{\xi}
\end{gathered}
$$

where $i$ denote the different species and $S_{m}, S_{M}, S_{h}$ and $S_{\xi}$ are source terms accounting for radiative effects, impact of liquid precursor on thermal flow or chemical reaction sources. According to how the turbulent viscosity, conductivity and species diffusion coefficient are modeled with a RANS or a LES approach, the variable $u, p, h$ and $e$ must be understood as statistical mean values for RANS turbulence models whereas these variables correspond to the large scale resolved filtered unknowns in the framework of LES. The turbulent coefficients $\mu_{t}, \lambda_{\mathrm{t}}$ and $D_{i, t}$ are generally obtained by means of a RANS statistical modeling. Reynolds decomposition is introduced for each variable which is split into an averaged statistical quantity and a fluctuating part. The closure assumptions are often based on the analogy of the Kolmogorov energy cascade [49], which is valid for thermal flows: the smallest scales of the momentum, enthalpy and mass fraction of species are dissipated into heat. Diffusive models applied to the unclosed turbulent terms lead to the introduction of turbulent viscosity, conductivity and species diffusion coefficients appearing in Equations (1) - (4). This system brings the local mean statistical pressure, velocity, mass fraction of species and temperature distributions that can be used to follow the trajectories and the temperature histories of precursor droplets injected within the flame by a Lagrangian integration scheme which reads:

$$
\begin{gathered}
m_{p} \frac{\mathrm{d} \boldsymbol{V}_{p}}{\mathrm{~d} t}=\boldsymbol{F}_{d}+\boldsymbol{F}_{a}+\boldsymbol{F}_{b} \\
m_{p} C_{p} \frac{\mathrm{d} T_{p}}{\mathrm{~d} t}=\pi d \lambda N u\left(T_{\mathrm{f}}-T_{p}\right)+\frac{\mathrm{d} m_{p}}{\mathrm{~d} t}\left|\boldsymbol{V}_{p}\right|+Q_{r} \\
\frac{\mathrm{d} r_{p}}{\mathrm{~d} t}=-\frac{\mathrm{d} m_{p}}{\mathrm{~d} t} \frac{1}{4 \pi \rho_{p} r_{p}^{2}}
\end{gathered}
$$

where $F_{D}, F_{b}$ and $F_{a}$ are respectively drag, buoyancy and additional mass forces whereas $Q_{r}$ is the radiative flux to which are subjected the liquid precursor particles. The model (1) - (7) has been used by many authors to characterize the behavior of various precursor droplets in HVOF and plasma suspension processes [5] [11] [26] [33]. Among them, typical obtained results are given in 
Figure 2. Concerning the operating conditions before the impact zone forming the final coating, important information such as the particle temperature or velocity can be estimated by means of the Lagrangian modeling. It can be remarked that $10 \%$ differences are observed compared to experiments. They are in particular due to the assumption of the modeling itself which only considers an isolated droplet in the simulation, which is not the case in real processes. To finish with, it has to be noticed that the model (5) - (7) has been used without a RANS representation of the mass, momentum, energy and mass fraction transfer
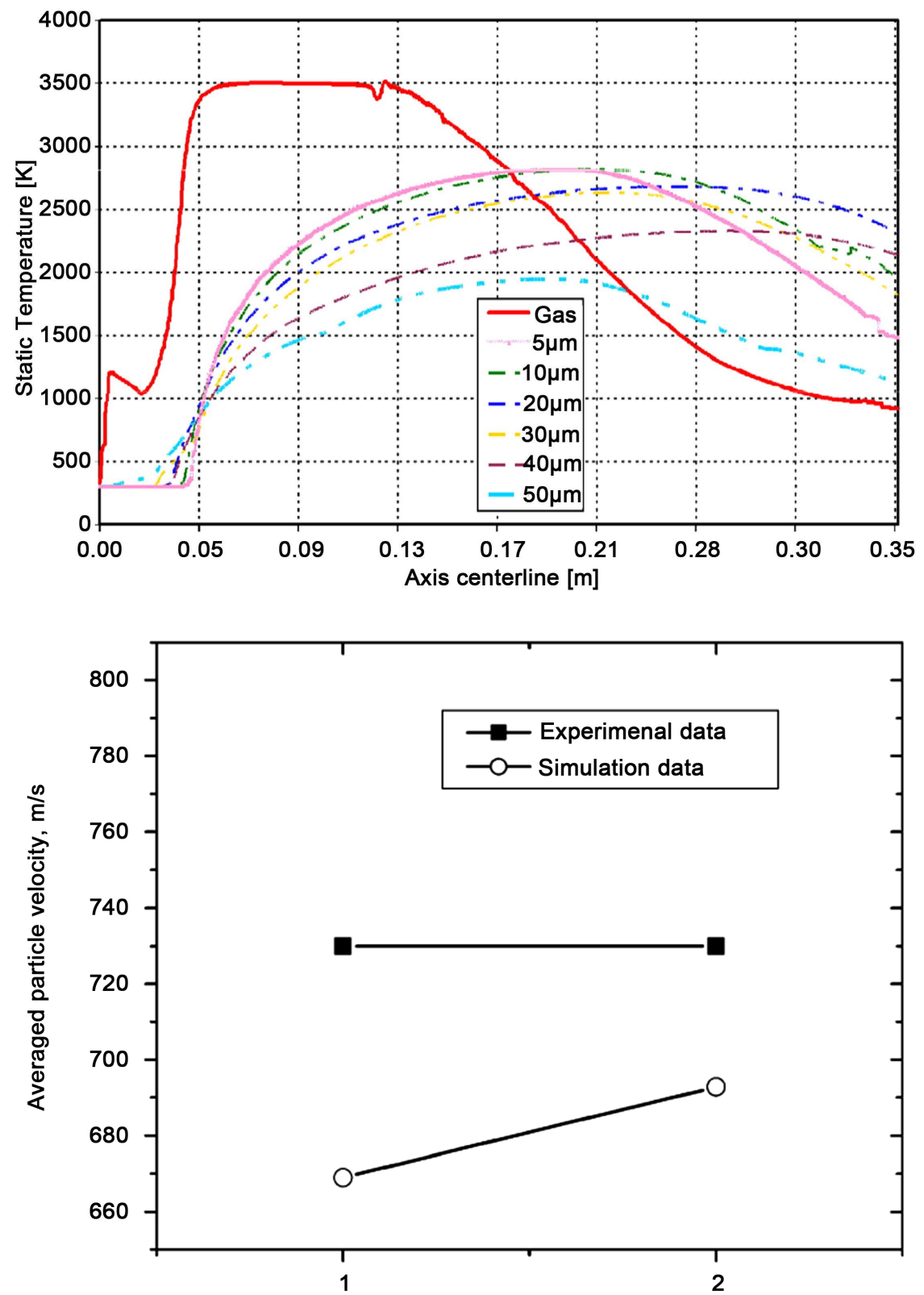

Figure 2. Typical results obtained with RANS modeling of the thermal flow and Lagrangian modeling of the particle tracking-Upper: temperature history of precursor droplets in a HVOF gun for various initial diameters [6]; Bottom: comparison between experiments and simulation of ethanol droplets of $50 \mu \mathrm{m}$ containing various sizes of zirconia and alumina nanoparticles (cases 1 and 2 respectively) [27]. 
(1) - (4) to also report the interaction between liquid precursor and thermal flow. For example, Basu and co-workers [50] [51] have studied the Lagrangian history of precursor droplets by using directly the mean temperature and velocity in a HVOF flame and by estimating the break-up events, heat transfer coefficients and vaporization through existing correlations of the literature. The developments of Marchand et al. [36] [52] are also interesting as they were the first to associate a deterministic LES representation of turbulence to simulate the interaction between a plasma flow and liquid precursor particles by a Lagrangian modeling of the droplets. They have studied for example the evolution of the Weber number of the liquid particles according to their initial radius [33]. These Weber numbers were used to characterize the secondary fragmentation modes of the precursor droplets. This LES turbulence modeling will be developed in the next section.

The main limitation of the Lagrangian modeling of liquid precursor/thermal flow interaction is a priori definition of interaction laws for the liquid particles with the surrounding fluid in terms of drag law, heat transfer coefficient or Nusselt number and vaporization mass transfer coefficients. These laws do not generally account for the presence of other droplets in the vicinity of the considered particle or their modification of the thermal flow (cooling effect, liquid break-up mode, deceleration, air engulfment...). In addition, only isolated liquid droplets are followed by the Lagrangian model, so that no coalescence or explicit secondary break-up is solved. The Lagrangian models can be improved by introducing two- or four-way coupling models for accounting of the effect of particles on the flame and also interaction between particles themselves [9]. These models are much more representative of real dispersed phases under denser conditions. However, they still rely on a scale separation between the size of the particles and the turbulent scales and they also need to define a priori particle-flow [26] and particle-particle interaction models which are difficult or even impossible to characterize experimentally. These limitations will be partly tackled with Eulerian approaches presented in the following section which are designed to solve a majority of time and space scales of the interfacial flow. The interest of Eulerian small scale modeling is to provide information for defining two- and four-way Lagrangian models in dense situations.

\section{Eulerian Models for Liquid Precursor Interaction with a Thermal Flow}

\subsection{Modeling}

The building of an Eulerian modeling for the interaction between a liquid precursor jet and a thermal flow aims at describing all the time and length scales of the liquid primary and secondary break-up under thermal flow shearing with thermal transfers and vaporization being solved at the same time. These objectives intrinsically rely on a deterministic description of the problem concerning liquid deformation and rupture, as well as turbulence structures. As a conse- 
quence, the previously presented RANS modeling of the thermal flow cannot be used in this description of the problem as each space scale of droplet generation and modification will be solved at each time step. The only work which reports on a compressible model for the simulation at small scale of the interaction between a precursor liquid jet with a plasma flow is based on the following model [15] [18] [34] [53]:

$$
\begin{gathered}
\frac{\partial p}{\partial t}+\frac{\tau}{\chi_{T}} \nabla \cdot \boldsymbol{u}=0 \\
\rho\left[\frac{\partial \boldsymbol{u}}{\partial t}+\boldsymbol{u} \cdot \nabla \boldsymbol{u}\right]=-\nabla\left(p-\frac{\tau}{\chi_{T}} \nabla \cdot \boldsymbol{u}\right)+\nabla \cdot\left(\mu+\mu_{t}\right)\left[\nabla \boldsymbol{u}+\nabla^{t} \boldsymbol{u}\right]+\boldsymbol{S}_{S T} \\
\rho C_{p}\left[\frac{\partial T}{\partial t}+\boldsymbol{u} \cdot \nabla T\right]=\nabla \cdot\left(\lambda+\lambda_{t}\right) \nabla T+S_{h} \\
\frac{\partial \chi}{\partial t}+\boldsymbol{u} \cdot \nabla \chi=\nabla \cdot\left(D+D_{t}\right) \nabla \chi+S_{\xi} \\
\frac{\partial C}{\partial t}+\boldsymbol{u} \cdot \nabla C=0
\end{gathered}
$$

where $\tau$ is a characteristic time of the problem chosen equal to the numerical time step $\Delta t$ used to discretize the time derivatives. The liquid volume fraction $\mathrm{C}$ is representative of the volume of liquid precursor in each elementary volume or grid cell. By definition, $C=1$ in the liquid and $C=0$ elsewhere. This approach is termed Volume of Fluid (VOF) method in the literature [54]. As explained in [34], the local characteristics $\rho, \mu, \lambda$ and $C_{p}$ of the fluids (air, plasma gas and liquid) are built according to the pressure, temperature, plasma gas concentration $\chi$ and liquid volume fraction $C$. The surface tension forces are determined according to $C$ and integrated as a local source term in the cells cut by the interface through a volume force $S_{S T}$ [34]. The vaporization of the liquid is not accounted for as the Eulerian model is adapted to modeling the first instant interaction between the liquid jet and the plasma. The main difference between the RANS model (1) - (4) and the deterministic model (8) - (12) is the modeling of turbulence. In the latter, a LES approach is used to build the turbulent viscosity $\mu_{t}$ [53] by assuming that the larger scales of the multi-phase flow are resolved while the smallest one are modeled by means of a diffusive behavior. Once $\mu_{t}$ is known, the turbulent conductivity $\lambda_{t}$ and plasma gas diffusion coefficient $D_{t}$ are obtained by means of a turbulent Prandtl and Lewis analogy. With model (8) - (12), it is assumed that all the time and space scales of the liquid precursor interface are solved, meaning that the discretization grid has to be refined enough to capture all the interfacial structures generated by the interaction of the liquid jet with the thermal flow. Due to heat exchange between water and plasma, the phase change cannot be neglected for long time simulations. A phase change model is integrated; it is an adaptation of a Lagrangian model of [55]. However, the reduction of liquid volume is considered while the motion of water vapor created during the evaporation is not taken into account in the simulations. 
The droplet diameter decreases according to the following law:

$$
d_{p}^{n+1}=d_{p}^{n}-\Delta t \frac{4 \lambda_{g}}{\rho_{p} c_{p, g} d_{p}^{n}} N u \ln \left(1+B_{T}\right)
$$

where $d_{p}$ is the drop diameter, $\Delta t$ the time step, $\lambda$ the thermal conductivity, $\rho$ the density, $c_{p}$ the specific heat, $N u$ the Nusselt number, $B_{T}$ the thermal Biot number and $n$ the discretization $t$ index.

Each cell cut by the interface is considered as an equivalent sphere whose tradius is calculated as the local curvature radius $\nabla \cdot \frac{\nabla C}{\|\nabla C\|}$. The Equation (1) is applied on these equivalent spheres. The evaporated water volume is calculated and removed in the concerned cells and finally the phase function $\mathrm{C}$ is updated. All variables are local and known in each cell of the mesh.

\subsection{Results}

Typical results obtained with the Eulerian LES model are presented in Figure 3. The calculation domain is restricted to the injection zone. Its dimensions are 4 $\mathrm{mm} \times 3.56 \mathrm{~mm} \times 1 \mathrm{~mm}$. The mesh is $410 \times 300 \times 130$, to fit approximately 16 million of cells. The time step is $\Delta \mathrm{t}=10^{-8} \mathrm{~s}$. The computations were performed with a Sulzer Metco PTF4 torch, with a nozzle diameter of $6 \mathrm{~mm}$, discharging Ar $\mathrm{H}_{2}$ into ambient air. The flow rate is $45 / 15 \mathrm{slm}$. The torch was assumed to operate at $500 \mathrm{~A}$ and $65 \mathrm{~V}$, with a thermal efficiency of 52\%. The velocity and temperature profiles are imposed at the domain entry, according to previous calculations of plasma flow with time-depending boundary conditions [56]. The velocity of the cross-flow, corresponding to peripheral velocity conditions in the plasma, is included between 0 and $850 \mathrm{~m} \cdot \mathrm{s}^{-1}$ and the temperature profile of the cross-flow is included between 3000 and $9000 \mathrm{~K}$ for $\mathrm{ArH}_{2}$ flow [16].

The primary and secondary fragmentations of the precursor jet can be observed and typical probability density functions of droplet size can be extracted from this type of simulation. The main drawback of the Eulerian modeling is that it is not possible to use a grid refined enough on existing parallel computers (512 processors are used in Figure 3 ) to capture the finest liquid droplets after fragmentation. In the presented simulation, the smallest resolved droplets have a diameter equal to $1 \mu \mathrm{m}$. The same conclusions apply when using a Lagrangian approach for solving the liquid-thermal flow interaction.

First of all, the interactions between the different fluids can be defined by the Weber number. Classically, the gas Weber number $\left(\mathrm{We}_{\mathrm{g}}\right)$ is defined as the ratio of the disrupting inertial forces to the restorative surface tension forces. Previous works (ref-CC2) were dedicated to the calculation of the Weber number versus the plasma flow radius. These ones show different break-up modes depending on the investigation zone. It was found that instability waves developed along the liquid shape in interaction with the plasma flow. These waves were the roots of threads which rapidly broke into big droplets in the first layers of the flow. Analyses of the Weber number versus the plasma flow radius showed its high 

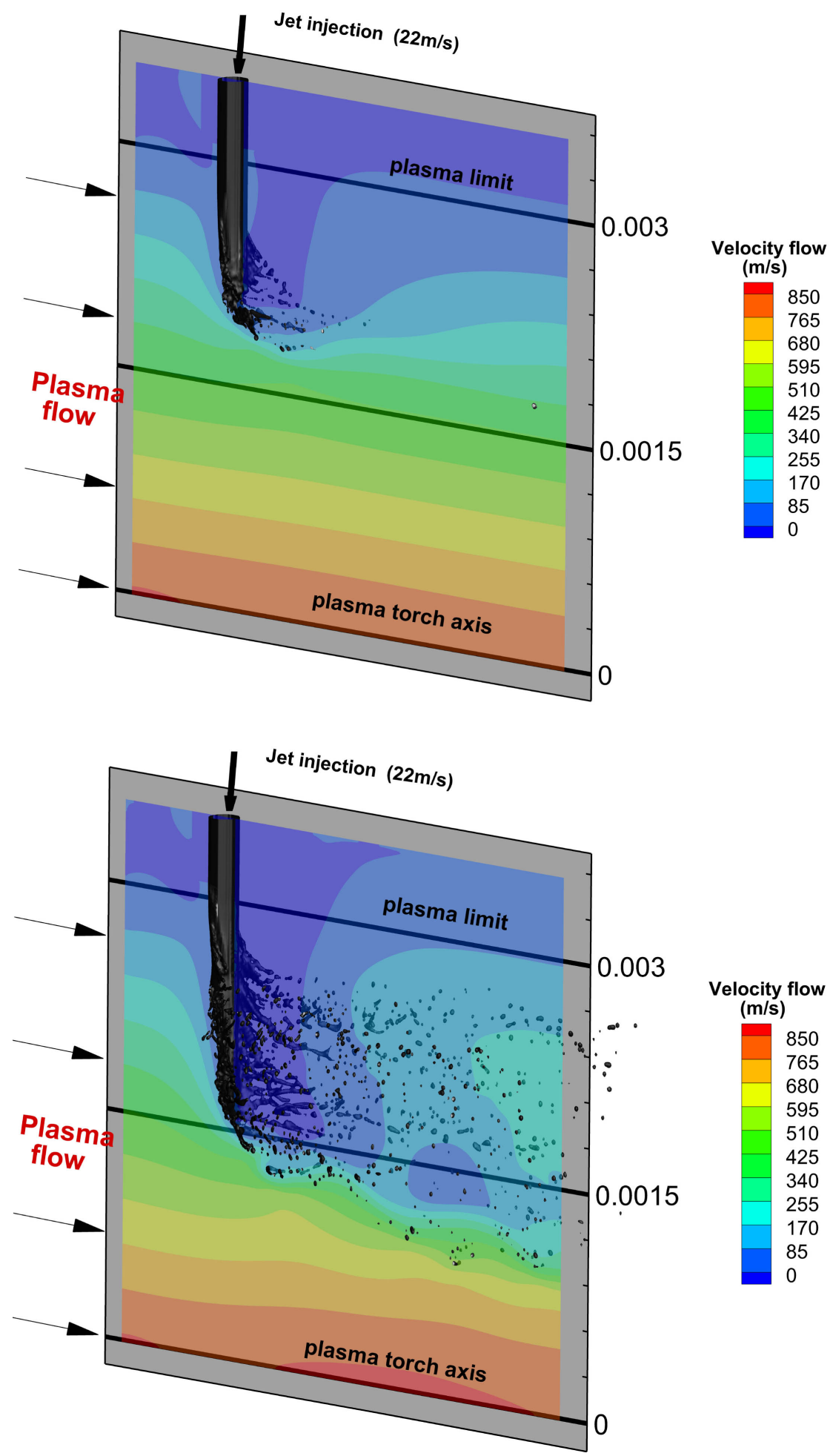

Figure 3. Injection of a liquid jet into an $\mathrm{ArH}_{2}$ plasma flow at $t=50 \times 10^{-6} \mathrm{~s}$ (Upper) and $t=100 \times 10^{-6} \mathrm{~s}$ (Bottom).

evolution due to the velocity and density gradients which, in contrast, moved one by one. This variation explains the complexity of the fragmentation and the unusual modes observed. 
The evolution of the jet injection into $\mathrm{ArH}_{2}$ plasma can be seen in Figure 3 . Two steps can be analyzed. The first one concerns the first instants (less than 50 $\mu \mathrm{s})$ during which the liquid column penetrates the plasma flow. The destruction of the liquid starts just at the tip of the column with appearing of ligaments after a short run in the flow: only one mm of run is necessary to create them. Simultaneously, instabilities appear at the liquid-shape surface and grow up to the close limit of the plasma jet (Bottom in Figure 3). Then the filaments break into droplets in the continuity. As seen in the second picture (Figure 3 Bottom), the balance is achieved in less than $100 \mu$ s. Instabilities expand on the jet surface and stay active as soon as the jet reaches the plasma zone (around $0.003 \mathrm{~mm}$ ). Now, a lot of ligaments are formed at the periphery of the jet and are stretched and broken up into large droplets (primary fragmentation). According to the low introduction pressure $(0.25 \mathrm{MPa})$ the penetration length is about $1.3 \mathrm{~mm}$ above the torch axis.

An overpressure zone is well observed in the upstream direction to the jet (Figure 4 Bottom).

The plasma temperature decreases strongly with the liquid injection testifying from air engulfment inside the plasma (Figure 4 Upper): puffs of cold air can be seen around big droplets flowing downstream.

Two calculations types have been done with and without phase change. To differentiate the droplet behavior and the impact of the phase change, the droplet number has been calculated at the same time $(100 \mu \mathrm{s})$ in the whole field. With and without the phase change model, the jet behavior does not seem different, but the droplet number decreases due to the water vaporization (Figure 5). The distribution of the droplet number according to their diameters is represented in Figure 5.

If the biggest size droplets stay in the same number range (more than $38 \mu \mathrm{m}$ ) due to their thermal inertia, the lowest diameter droplets diminish in number because of the evaporation. The lost mass can be estimated to $10 \%$ that is not negligible in a so short time, less than $50 \mu$ s, required time to get the system in balance.

The domain is cut in several parts from the center line to the border of the plasma jet (Figure 6 according to [57]) in order to determine the droplet distribution along the plasma radius.

It is observed that smaller droplets $(10 \mu \mathrm{m})$ are especially present in the zones close to the torch axis. In this place, temperature and velocity are higher; thus droplets are more evaporated or broken-up. On the contrary, droplets with a diameter of the order of $30 \mu \mathrm{m}$ are more numerous in the peripheral zone (Figure 7).

\section{Mixed Eulerian-Lagrangian Models for the Interaction between a Thermal Flow and a Liquid Precursor}

As recommended by Cetegen and Basu [9], with the advances in parallel computational facilities and understanding of models, multi-scale models are 

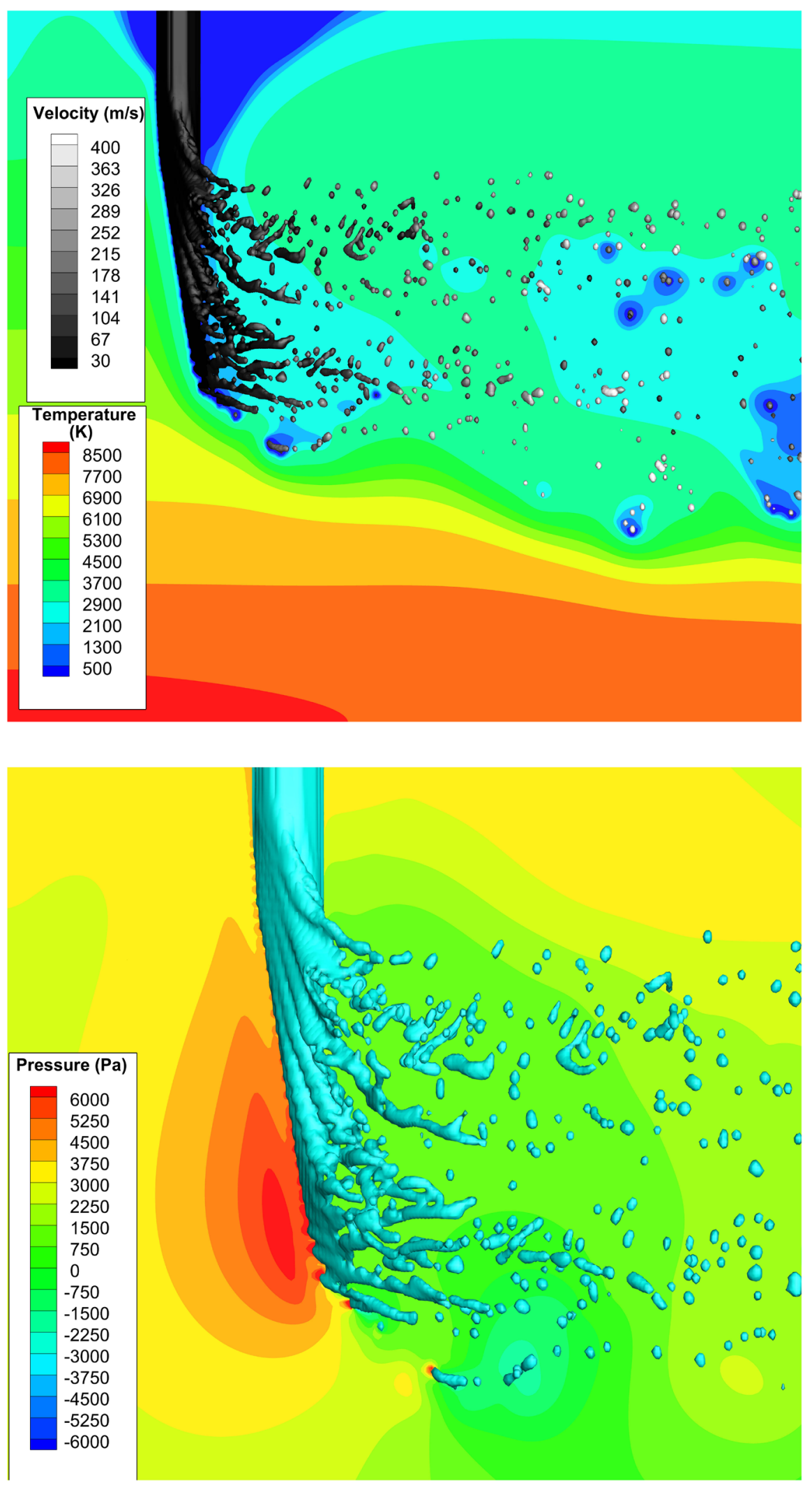

Figure 4. Injection of a liquid jet into an $\mathrm{ArH}_{2}$ plasma flow at $t=100 \times 10^{-6} \mathrm{~s}$. (Left: Temperature in a slice along the torch axis and velocity of the water droplets. Right: Pressure in a slice along the torch axis). 


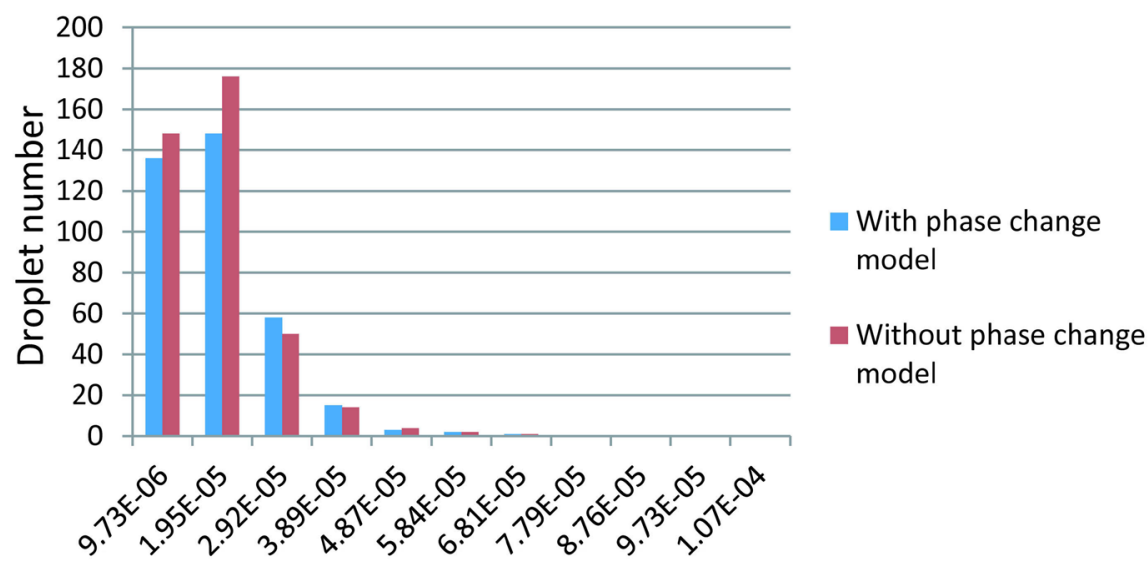

Diameter $(\mathrm{m})$

Figure 5. Distribution of droplet in the calculation domain with and without phase change model at $t=100 \times 10^{-6} \mathrm{~s}$.

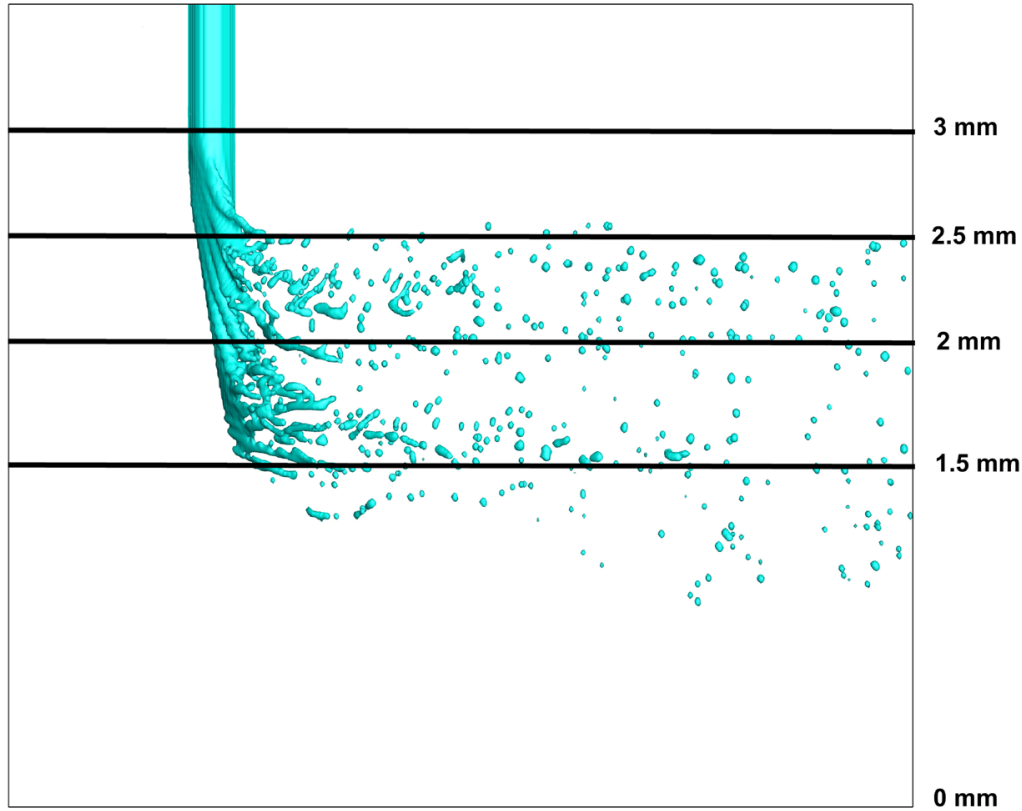

Figure 6. Radial zones used for the calculation of the droplet size distribution.

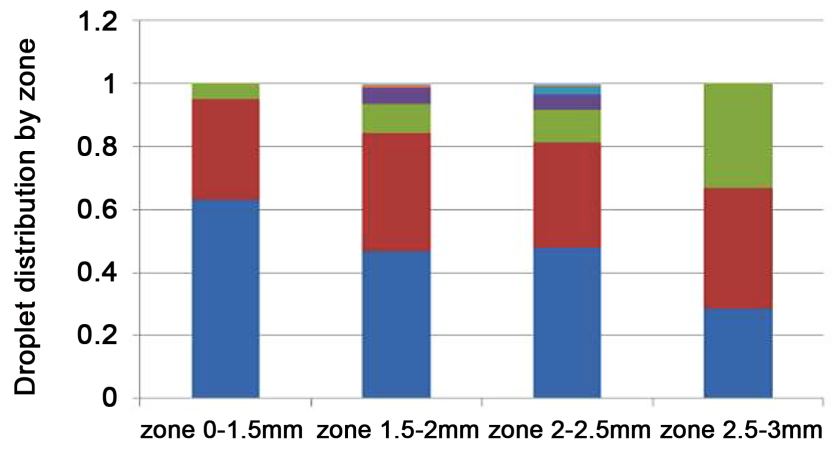

Diameter (m)

6.81E-05

- 5.84E-05

4.87E-05

- 3.89E-05

2.92E-05

$-1.95 \mathrm{E}-05$

9.73E-06

Figure 7. Droplet distribution according to the diameter by radial zone in the calculation domain. 
envisaged as efficient tools in understanding the thermal spray process in the near future. In this section, a proposition is made to build efficient and realistic multi-scale models for thermal spray processes. By considering the general structure of a typical plasma-liquid jet interaction, as presented in Figure 8, it can be first observed that a large range of interfacial scales are generated by this interaction. Secondly that the smallest liquid droplet remains spherical due to surface tension effects and finally that after a certain duration (or distance) into the flame outlet, no fragmentation occurs, leading to a convergence of the droplet size (before vaporization). Solving all the phases of the liquid-flame interaction requires to describe, using the same model, the primary and secondary fragmentations of the jet (Eulerian LES modeling) as well as the time history of small liquid droplets with vaporization (Lagrangian modeling is suitable in this case as the physical phenomena are well known). In this way, the effects of unsteadiness coming from the torch will interact with the modes of jet fragmentation and also influence the final liquid droplet distribution. By solving at the same time the precursor droplet histories and the surrounding plasma flow and heat transfers, the instantaneous dynamics of the problem will be obtained. This is a very important aspect of the modeling as the initial positions of the liquid precursor droplets is not a priori known. The initial position of precursor droplets and resulting initial position of ceramic nanoparticles will not be decided by the user but by the simulated secondary fragmentation of the liquid jet.

It is proposed to use a deterministic LES compressible model (8) - (12) everywhere on a computational grid whose finest grid cell is of the size of the smaller droplets in the secondary break-up zone (namely $d_{d 1}$ in Figure 8). As

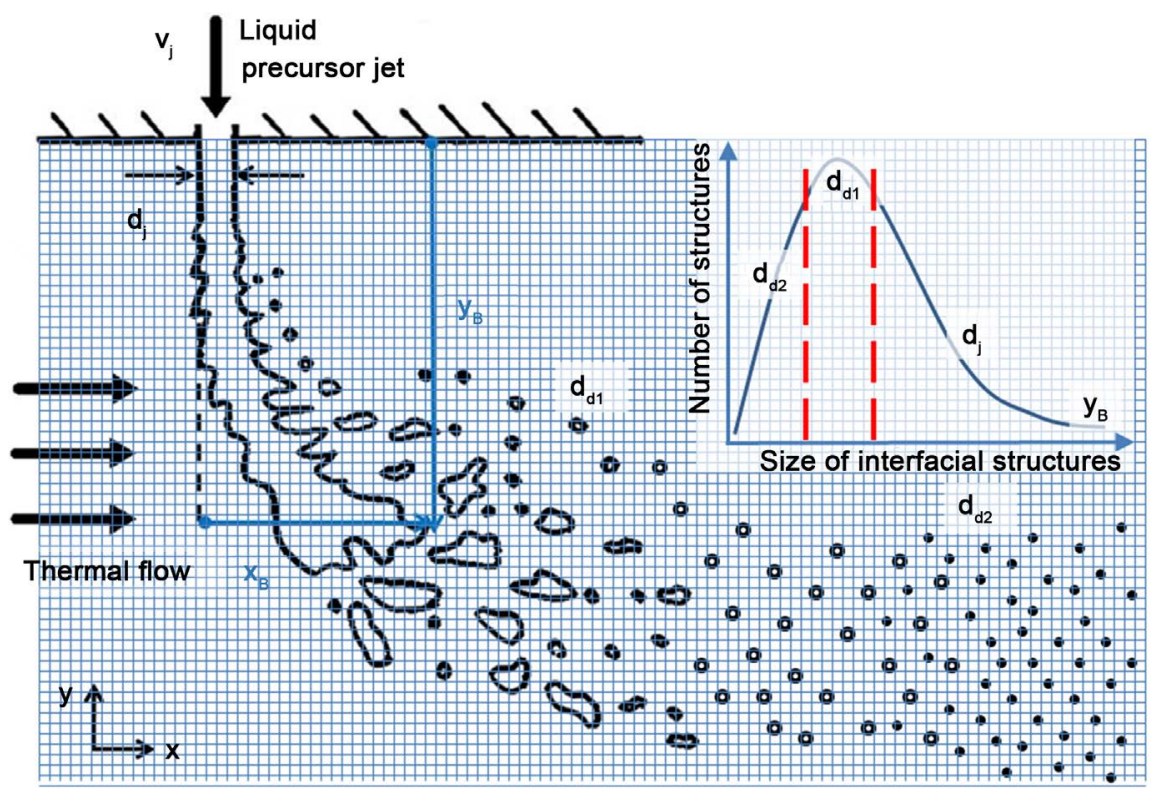

Figure 8. Scheme of liquid precursor jet interaction with a thermal flow-Representation of a typical Eulerian computational grid and the related pdf of particle sizes. The red dotted lines describe the zone of smaller resolved interfacial scales by means of Eulerian model (8) - (12). 
soon as a liquid droplet diameter is smaller than $d_{d 1}$, namely range $d_{d 2}$, the local VOF function $\mathrm{C}$ is chosen equal to 0 in the Eulerian model and these particles are then considered as a Lagrangian object to which Lagrangian macroscopic physical models for velocity, radius, temperature and vaporization, similar to those described in (5) - (7), are applied. The correct numerical method for implementing such a multi-scale Eulerian-Lagrangian model is the VOF-Sub Mesh approach [54]. Indeed, this method can describe at the same time an Eulerian VOF function $\mathrm{C}$ and also Lagrangian markers (Lagrangian droplets in our multi-scale model) whose volume, velocity and temperature can be modeled differently to those applied to $C$. The first implementation of the VOF-SM multi-scale model, including Lagrangian modeling of the droplet vaporization (with diameters in the range $\mathrm{d}_{\mathrm{d} 2}$ ), has been first presented by Caruyer et al. [58]. With the VOF-PLIC method [59], the droplet size cannot be inferior to the cell size. It implies the artificial generation of large liquid precursor droplets to conserve the liquid volume over time and thus a truncation of the droplet distribution. In order to counter that, the new hybrid method VOF-SM [57], can track the sub-mesh interface by means of Lagrangian markers. As seen in Figure 9, with the VOF-PLIC method, larger droplets $(>20 \mu \mathrm{m})$ are overestimated, as expected, to the detriment of the smallest.

\section{Conclusions and Prospects}

Thermal spray technologies, such as HVOF or plasma spraying, are able to produce nano structured coatings by introducing nano material suspensions into the high temperature and high velocity gas flow. The high radiation and velocity of the flow complicate the analysis of the phenomenon. Hence, numerical investigations can give numerous information of interest. From this review, the following aspects should be integrated in a Computational Fluid Dynamics code to achieve elementary or global simulations:

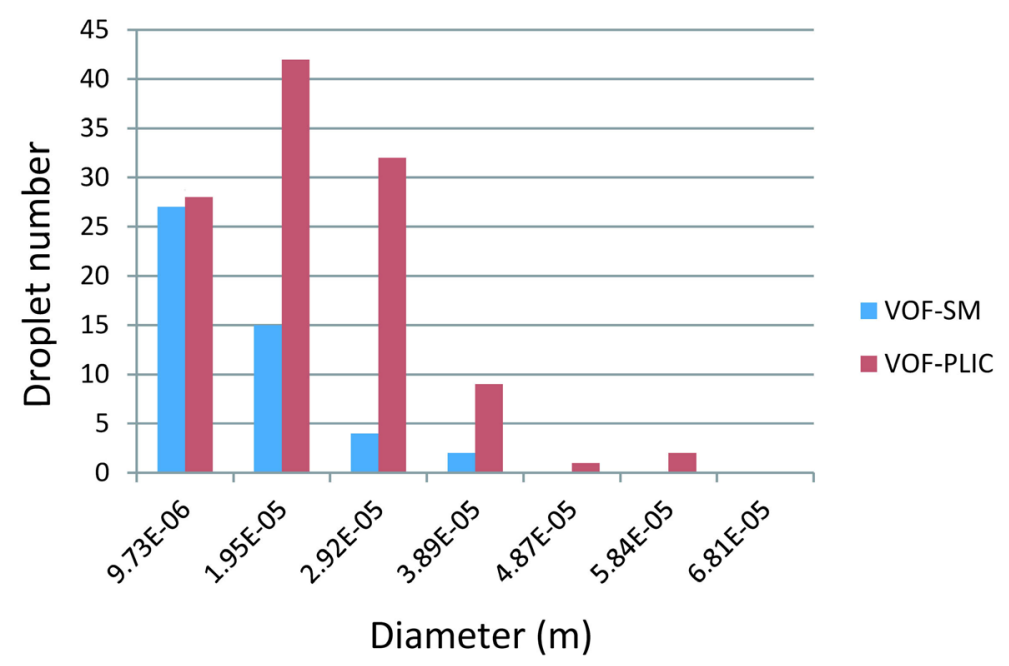

Figure 9. Distribution of droplet in the whole calculation domain with VOF-PLIC and VOF-SM interface tracking methods at $t=50 \times 10^{-6} \mathrm{~s}$. 
- Compressible effects of the flow,

- High resolution of the turbulence with LES turbulence models and large interfacial scales,

- High number of physical identities (solid particle, droplet) treated by Eulerian methods.

In fact, the interactions between the liquid and high velocity and high temperature gas flows, such as those generated by HVOF or DC plasma guns, lead to such a large range of droplet sizes, size which evolves with time during evaporation, that the taking-into-account of numerous and small physical entities can only be treated by mixed Eulerian-Lagrangian models. That is the main strategy proposed here as conclusion and advocated by the authors to analyze in depth the behavior of nanoparticles injected in thermal spray processes.

\section{Acknowledgements}

The authors thank the Aquitaine Regional Council for the financial support dedicated to a 256-processor cluster investment. We are grateful for access to the computational facilities of the French CINES (National computing center for higher education) under project number A0012b06115 and CCRT (Research and Technology Computing Center) under project number gen6115. The authors also thank the CEA for its support. The ANR Modemi is also associated to this project concerning multi-scale modeling of multi-phase flows.

\section{References}

[1] Gitzhofer, F., Bouyer, E. and Boulos, M.I. (1997) Suspension Plasma Spraying. U.S. Patents, 5, 609, 921.

[2] Karthikeyan, J.J., Berndt, C.C., Tikkanen, J., Reddy, S. and Herman, H. (1997) Plasma Spray Synthesis of Nanomaterial Powders and Deposits. Surface and Coatings Technology, 238, 275-286.

[3] Karthikeyan, J.J., Berndt, C.C., Reddy, S., Wang, J.Y., King, A.H. and Herman, H. (1998) Nanomaterials Deposits Formed by dc Plasma Spraying of Liquid Feedstocks. Journal of the American Ceramic Society, 81, 121-128. https://doi.org/10.1111/j.1151-2916.1998.tb02303.x

[4] Bouyer, E., Gitzhofer, F. and Boulos, M. (1996) Parametric Study of Suspension Plasma Sprayed Hydroxyapatite. In: Berndt, C.C., Ed., Proceedings of the ITSC, ASM International, Almere, 683-691.

[5] Dongmo, E., Gadow, R., Killinger, A. and Wenzelburger, M. (2009) Modeling of Combustion as Well as Heat, Mass, and Momentum Transfer during Thermal Spraying by Hvof and Hvsfs. Journal of Thermal Spray Technology, 18, 896-908. https://doi.org/10.1007/s11666-009-9341-2

[6] Fauchais and Montavon, G. (2010) Latest Developments in Suspension and Liquid Precursor Thermal Spraying. Journal of Thermal Spray Technology, 19, 226-239. https://doi.org/10.1007/s11666-009-9446-7

[7] Fauchais, P. and Vardelle, A. (1994) Plasma Spraying: Present and Future. Pure \& Applied Chemistry, 66, 1247-1258. https://doi.org/10.1351/pac199466061247

[8] Wittmann-Ténèze, K. (2001) Etude de l'élaboration de couches minces par 
projection plasma. PhD Thesis, Université de Limoges, Limoges. (In French)

[9] Cetegen, B.M. and Basu, M. (2009) Review of Modeling of Liquid Precursor Droplets and Particles Injected into Plasmas and High-Velocity Oxy-Fuel (Hvof) Flame Jets for Thermal Spray Deposition Applications. Journal of Thermal Spray Technology, 18, 769-793. https://doi.org/10.1007/s11666-009-9365-7

[10] Killinger, A., Gadow, R., Mauer, G., Guignard, A., Vaßen, R. and Stöver, D. (2011) Review of New Developments in Suspension and Solution Precursor Thermal Spray Processes. Journal of Thermal Spray Technology, 20, 677-695. https://doi.org/10.1007/s11666-011-9639-8

[11] Dongmo, E., Wenzelburger, M. and Gadow, R. (2008) Analysis and Optimization of the Hvof Process by Combined Experimental and Numerical Approaches. Surface and Coating Technology, 202, 4470-4478.

https://doi.org/10.1016/j.surfcoat.2008.04.029

[12] Fincke, J.R., Crawford, D.M., Snyder, S.C., Swank, W.D., Haggard, D.C. and Williamson, R.L. (2003) Entrainment in High-Velocity, High-Temperature Plasma Jets. Part I: Experimental Results. International Journal of Heat Transfer, 46, 4201-4213. https://doi.org/10.1016/S0017-9310(03)00272-2

[13] Duan, Z. and Heberlein, J. (2002) Arc Instabilities in a Plasma Spray Torch. Journal of Thermal Spray Technology, 11, 44-57. https://doi.org/10.1361/105996302770348961

[14] Coudert, J.F., Planche, M.P. and Fauchais, P. (1995) Velocity Measurement of dc Plasma Jets Based on Arc Root Fluctuations. Plasma Chemical Plasma Process, 15, 47-70. https://doi.org/10.1007/BF01596681

[15] Meillot, E., Vincent, S., Caruyer, C., Caltagirone, J. and Damiani, D. (2009) From dc Time Dependent Thermal Plasma Generation to Suspension Plasma-Spraying Interactions. Journal of Thermal Spray Technology, 18, 875-886.

https://doi.org/10.1007/s11666-009-9354-x

[16] Caruyer, C., Vincent, S., Meillot, E., Caltagirone, J. and Damiani, D. (2010) Analysis of the Unsteadiness of a Plasma Jet and the Related Turbulence. Surface and Coating Technology, 205, 1165-1170. https://doi.org/10.1016/j.surfcoat.2010.07.025

[17] Fauchais, P., Etchart-Salas, R., Delbos, C., Tognonvi, M., Rat, V., Coudert, J.F. and Chartier, T. (2007) Suspension and Solution Plasma Spraying of Finely Structured Layers: Potential Application to SOFCs. Journal of Physics D: Applied Physics, 40, 2394-2406. https://doi.org/10.1088/0022-3727/40/8/S19

[18] Caruyer, C., Vincent, S., Meillot, E., Damiani, D. and Caltagirone, J. (2011) Effects of Plasma Conditions on the Fragmentation and the Atomization of a Liquid Jet. Proceedings of the 24th European Conference on Liquid Atomization and Spray Systems ILASS, Estoril, September 2011.

[19] Rayleigh, L. (1879) On the Capillary Phenomena of Jets. Proceedings of the Royal Society of London, 29, 71-97. https://doi.org/10.1098/rspl.1879.0015

[20] Pilch, M. and Erdman, C.A. (1987) Use of Breakup Time Data and Velocity History Data to Predict the Maximum Size of Stable Fragments for Acceleration-Induced Breakup of a Liquid Drop. International Journal of Multiphase Flow, 13, 741-757. https://doi.org/10.1016/0301-9322(87)90063-2

[21] Ranz, W. and Marshall, W. (1952) Evaporation from Drops. Chemical Engineering Progress, 48, 141-146.

[22] Chen, X. (1988) Particle Heating in a Thermal Plasma. Pure and Applied Chemistry, 60, 651-662. https://doi.org/10.1351/pac198860050651 
[23] O'Rourke, P.J. and Amsden, A.A. (1987) The Tab Method for Numerical Calculation of Spray Droplet Breakup. SAE Technical Paper.

[24] Mariaux, G. and Vardelle, A. (2005) 3-D Time-Dependent Modelling of the Plasma Spray Process. Part 1: Flow Modeling. International Journal of Thermal Science, 44, 357-366. https://doi.org/10.1016/j.ijthermalsci.2004.07.006

[25] Sirignano, W.A. (1999) Fluid Dynamics and Transport of Droplets and Sprays. Cambridge University Press, New York. https://doi.org/10.1017/CBO9780511529566

[26] Shan, Y., Coyle, T.W. and Mostaghimi, J. (2007) Numerical Simulation of Droplet Breakup and Collision in the Solution Precursor Plasma Spraying. Journal of Thermal Spray Technology, 16, 698-704. https://doi.org/10.1007/s11666-007-9102-z

[27] Ozturk, A. and Cetegen, B.M. (2005) Modeling of Axially and Transversely Injected Precursor Droplets into a Plasma Environment. International Journal of Heat and Mass Transfer, 48, 4367-4383. https://doi.org/10.1016/j.ijheatmasstransfer.2005.05.015

[28] Murphy, A.B. (1994) Transport Coefficient of Air, Argon-Air, Nitrogen-Air and Oxygen-Air Plasmas. Plasma Chemistry Plasma Process, 15, 279-307. https://doi.org/10.1007/BF01459700

[29] Boulos, M.I., Fauchais, P. and Pfender, E. (1994) Thermal Plasma, Fundamental and Application. Vol. 1, Plenum Press, New York and London.

[30] Selvan, B., Ramachandran, K., Pillai, B.C. and Subhakar, D. (2001) Numerical Modelling of ar-n2 Plasma Jet Impinging on a Flat Substrate. Journal of Thermal Spray Technology, 20, 534-548. https://doi.org/10.1007/s11666-010-9587-8

[31] Selvan, B., Ramachandran, K., Sreekumar, K., Thiyagarajan, T.K. and Ananthapadmanabhan, P.V. (2010) Numerical and Experimental Studies on dc Plasma Spray Torch. Vacuum, 84, 444-452.

https://doi.org/10.1016/j.vacuum.2009.09.009

[32] Williamson, R.L., Fincke, J.R., Crawford, D.M., Snyder, S.C., Swank, W.D. and Haggard, D.C. (2003) Entrainment in High-Velocity, High-Temperature Plasma Jets. Part II: Computational Results and Comparison to Experiment. International Journal of Heat Transfer, 46, 4215-4228. https://doi.org/10.1016/S0017-9310(03)00271-0

[33] Xiong, H.-B. and Lin, J.-Z. (2009) Nanoparticles Modeling in Axially Injection Suspension Plasma Spray of Zirconia and Alumina Ceramics. Journal of Thermal Spray Technology, 18, 887-895. https://doi.org/10.1007/s11666-009-9349-7

[34] Vincent, S., Balmigère, G., Caruyer, C., Meillot, E. and Caltagirone, J. (2009) Contribution to the Modeling of the Interaction between a Plasma Flow and a Liquid Jet. Surface and Coating Technology, 203, 2162-2171. https://doi.org/10.1016/j.surfcoat.2008.11.009

[35] Janisson, S., Meillot, E., Vardelle, A., Coudert, J.F., Pateyron, B. and Fauchais, P. (1999) Plasma Spraying Using Ar-He- $\mathrm{H}_{2}$ Gas Mixtures. Journal of Thermal Spraying Technology, 8, 545-552. https://doi.org/10.1361/105996399770350232

[36] Marchand, C., Chazelas, C., Mariaux, G. and Vardelle, A. (2007) Liquid Precursor Plasma Spraying: Modeling the Interactions between the Transient Plasma Jet and the Droplets. Journal of Thermal Spray Technology, 16, 705-712. https://doi.org/10.1007/s11666-007-9112-x

[37] Williamson, R.L., Fincke, J.R. and Chang, C.H. (2000) A Computational Examination of the Sources of Statistical Variances in Particle Parameters during Thermal 
Plasma Spraying. Plasma Chemistry Plasma Process, 20, 299-324. https://doi.org/10.1023/A:1007088110319

[38] Kai, C., Chen, X. and Pan, W.X. (2006) Comparison of Laminar and Turbulent Thermal Plasma Jet Characteristics. A Modeling Study. Plasma Chemistry Plasma Process, 26, 211-235. https://doi.org/10.1007/s11090-006-9006-6

[39] Trelles, J., Chazelas, C., Vardelle, A. and Heberlein, J.V.R. (2009) Arc Plasma Torch Modeling. Journal of Thermal Spray Technology, 18, 728-752. https://doi.org/10.1007/s11666-009-9342-1

[40] Moreau, E., Chazelas, C., Mariaux, G. and Vardelle, A. (2006) Modeling the Restrike Mode Operation of a DC Plasma Spray Torch. Journal of Thermal Spray Technology, 15, 524-530. https://doi.org/10.1361/105996306X147306

[41] Lebouvier, A., Delalondre, C., Fresnet, F., Boch, V., Rohani, V., Cauneau, F. and Fulcheri, L. (2011) Three-Dimensional Unsteady MHD Modeling of a Low-Current High-Voltage Non-Transferred DC Plasma Torch Operating with Air. IEEE Transactions on Plasma Science, 39, 1889-1899. https://doi.org/10.1109/TPS.2011.2160208

[42] Colombo, V., Concetti, A. and Ghedini, E. (2008) Three-Dimensional Time-Dependent Modeling of a DC Transferred Arc Twin-Torch System. IEEE Transactions on Plasma Science, 36, 1038-1039. https://doi.org/10.1109/TPS.2008.924501

[43] Huang, R., Fukanuma, H., Uesugi, Y. and Tanaka, Y. (2012) Simulation of Arc Root Fluctuation in a DC Non-Transferred Plasma Torch with Three Dimensional Modeling. Journal of Thermal Spray Technology, 21, 636-643.

https://doi.org/10.1007/s11666-011-9710-5

[44] Huang, R., Fukanuma, H., Uesugi, Y. and Tanaka, Y. (2013) Comparisons of Two Models for the Simulation of a DC Arc Plasma Torch. Journal of Thermal Spray Technology, 22, 183-191. https://doi.org/10.1007/s11666-012-9860-0

[45] Meillot, E., Guenadou, D. and Bourgeois, C. (2008) Three-Dimension and Transient D.C. Plasma Flow Modeling. Plasma Chemistry Plasma Process, 28, 69-84. https://doi.org/10.1007/s11090-007-9108-9

[46] Jadidi, M., Moghtadernejad, S. and Dolatabadi, A. (2016) Numerical Modeling of Suspension HVOF Spray. Journal of Thermal Spray Technology, 25, 451-464. https://doi.org/10.1007/s11666-015-0364-6

[47] Taleby, M. and Hossainpour, S. (2012) Numerical Investigation of High Velocity Suspension Flame Spraying. Journal of Thermal Spray Technology, 21, 1163-1172. https://doi.org/10.1007/s11666-012-9802-x

[48] Fauchais, P. and Vardelle, A. (2000) Pending Problems in Thermal Plasmas and Actual Development. Plasma Physics and Controlled Fusion, 42, 365-383. https://doi.org/10.1088/0741-3335/42/12B/327

[49] Kolmogorov, A.N. (1941) The Local Structure of Turbulence in Incompressible Viscous Fluid for Very Large Reynolds Numbers. Proceedings. Mathematical and Physical Sciences, 434, 9-13.

[50] Basu, S. and Cetegem, B.M. (2008) Modeling of Liquid Ceramic Precursor Droplets in a High Velocity Oxy-Fuel Flame Jet. Acta Materiala, 56, 2750-2759. https://doi.org/10.1016/j.actamat.2008.02.007

[51] Basu, S., Jordan, E.H. and Cetegem, B.M. (2008) Fluid Mechanics and Heat Transfer of Liquid Precursor Droplets Injected into High-Temperature Plasmas. Journal of Thermal Spray Technology, 17, 60-72. https://doi.org/10.1007/s11666-007-9140-6 
[52] Marchand, C., Vardelle, A., Mariaux, G. and Lefort, P. (2008) Liquid Precursor Plasma Spraying: Modeling the Interactions between the Transient Plasma Jet and the Droplets. Surface and Coating Technology, 202, 4458-4464. https://doi.org/10.1016/j.surfcoat.2008.04.027

[53] Vincent, S., Meillot, E., Caruyer, C., Damiani, D. and Caltagirone, J. (2010) Modeling and Simulation of Unsteady Turbulent Flows in Thermal Plasma Spraying Processes. In: Marcuso, R.J., Ed., Turbulence: Theory, Types and Simulation (Physics Research and Technology), Nova Publishing, Hauppauge, 551-568.

[54] Vincent, S., Balmigère G., Caruyer, C., Caltagirone, J. and Meillot, E. (2010) Eulerian/Lagrangian Multiscale Methods for Solving Scalar Equations Application to Incompressible Two-Phase Flows. Journal of Computational Physics, 229, 73-106. https://doi.org/10.1016/j.jcp.2009.09.007

[55] Kuo, K.K.Y. (1986) Principles of Combustion. John Wiley and Sons, New York.

[56] Caruyer, C., Vincent, S., Meillot, E. and Caltagirone, J. (2010) Modeling the First Instant of the Interaction between a Liquid and a Plasma Jet with a Compressible Approach. Surface and Coating Technology, 205, 974-979.

https://doi.org/10.1016/j.surfcoat.2010.07.073

[57] Meillot, E., Vincent, S., Caruyer, C., Damiani, D. and Caltagirone, J. (2013) Modelling the Interactions between a Thermal Plasma Flow and a Continuous Liquid Jet in a Suspension Spraying Process. Journal of Physics D: Applied Physics, 46, Article ID: 224017.

[58] Caruyer, C., Vincent, S., Meillot, E. and Caltagirone, J. (2011) Modeling of the Fragmentation of a Water Jet in the Liquid Precursor Plasma Spraying. International Workshop on Suspension and Solution Plasma Spraying S2 TS, Tours, 3-5 October 2011, No. 1090 .

[59] Lopez, J., Hernandez, J., Gomez, P. and Faura, F. (2005) An Improved PLIC-VOF Method for Tracking Thin Fluid Structures in Incompressible Two-Phase Flows. Journal of Computational Physics, 208, 51-74. https://doi.org/10.1016/j.jcp.2005.01.031 


\section{Nomenclature}

$\chi \quad$ Plasmagen gas concentration

$\chi_{T}$ Isothermal compressibility $\left(\mathrm{Pa}^{-1}\right)$

$\mu \quad$ Dynamic viscosity (Pa.s)

$\mu_{t} \quad$ Turbulent viscosity (Pa.s)

$\lambda$ Thermal conductivity $\left(\mathrm{W} \cdot \mathrm{m}^{-1} \cdot \mathrm{K}^{-1}\right)$

$\lambda_{t}$ Turbulent conductivity $\left(\mathrm{W} \cdot \mathrm{m}^{-1} \cdot \mathrm{K}^{-1}\right)$

$\rho$ Density $\left(\mathrm{Kg} \cdot \mathrm{m}^{-3}\right)$

$\tau$ Characteristic time (s)

$\xi \quad$ Mass fraction of species

$C$ Liquid volume fraction

$C_{p}$ Heat capacity $\left(\mathrm{J} \cdot \mathrm{kg}^{-1}\right)$

$d$ Liquid precursor diameter (m)

$D_{i}$ Molecular diffusion coefficient $\left(\mathrm{m}^{2} \cdot \mathrm{s}^{-1}\right)$

$D_{t}$ Turbulent diffusion coefficient $\left(\mathrm{m}^{2} \cdot \mathrm{s}^{-1}\right)$

$H \quad$ Enthalpy $\left(\mathrm{J} \cdot \mathrm{kg}^{-1} \cdot \mathrm{K}^{-1}\right)$

$m_{p}$ Mass of liquid precursor particle $(\mathrm{Kg})$

Ma Mach number

$N u$ Nusselt number

$p$ Pressure $(\mathrm{Pa})$

$t$ Time (s)

$r_{p} \quad$ Radius of a liquid precursor particle (m)

Re Reynolds number

$T$ Temperature (K)

$T_{f}$ Thermal flow temperature (K)

$T_{p}$ Liquid precursor temperature (K)

$U$ Velocity $\left(\mathrm{m} \cdot \mathrm{s}^{-1}\right)$

$V_{p}$ Liquid precursor velocity $\left(\mathrm{m} \cdot \mathrm{s}^{-1}\right)$

We Weber number 